\title{
Epidemiological and clinical characteristics of suspected COVID-19 patients in the isolation ward in Guangzhou, China: a cohort study
}

\author{
Shaoqiang Li", Zhengtu Li", Liyan Chen", Jing Cheng", Yangqing Zhan, Feng Yang, Qian Jiang, \\ Xiaofen Su, Yingying Xie, Zhile Wu, Wenliang Guo, Weili Gu, Nanshan Zhong, Shiyue Li, Feng Ye \\ State Key Laboratory of Respiratory Disease, National Clinical Research Center for Respiratory Disease, Guangzhou Institute of Respiratory \\ Health, the First Affiliated Hospital of Guangzhou Medical University, Guangzhou, China \\ Contributions: (I) Conception and design: F Ye; (II) Administrative support: N Zhong, S Li; (III) Provision of study materials or patients: Z Li, J \\ Cheng, L Chen; (IV) Collection and assembly of data: Y Zhan, F Yang, X Su, Y Xie, Z Wu, W Guo, W Gu; (V) Data analysis and interpretation: Q \\ Jiang; (VI) Manuscript writing: All authors; (VII) Final approval of manuscript: All authors. \\ \#These authors contributed equally to this work. \\ Correspondence to: Prof. Feng Ye. The First Affiliated Hospital of Guangzhou Medical University of Guangzhou Medical University, 151 Yanjiang Xi \\ Road, Guangzhou 510120, China. Email: tu276025@gird.cn.
}

Background: Since there are reports of cases of 2019-coronavirus disease (COVID-19) asymptomatic carriers in China recently and fever is one of the main symptoms, we aimed to distinguish COVID-19 cases from other febrile patients with clinical examinations in this study.

Methods: A total of 134 suspected COVID-19 patients in the isolation ward of the First Affiliated Hospital of Guangzhou Medical University were recruited from January 23 to May 23, 2020. We analyze the pathogenic form and clinical characteristics.

Results: Among them, pathogens were identified in only 84 patients (62.7\%), including $23(17.1 \%)$ with severe acute respiratory syndrome coronavirus-2 (SARS-CoV-2), 30 (22.3\%) with other viruses, 31 $(25.0 \%)$ with other pathogens and $3(3.5 \%)$ with mixed infections. The commonly observed symptoms of COVID-19 patients were cough, fever, fatigue, and muscle aches, which were significantly different than the symptoms of nonviral infections $(\mathrm{P}<0.05)$ but from those of other viral infections $(\mathrm{P}>0.05)$. Furthermore, lactate dehydrogenase and the neutrophil/lymphocyte were found significantly high in COVID-19 patients compared to non-COVID-19 patients $(\mathrm{P}<0.05)$. The most common manifestations of COVID-19 patients were ground-glass opacities (100\%) with or without lung consolidation, however, they also often showed involvement of several lobes of both lungs $(\mathrm{P}<0.05)$. Due to the clear differential diagnosis, the overall antibiotic use rate was $35.8 \%(31 / 87)$.

Conclusions: When diagnosing COVID-19, infections with other pathogens should not be ignored. Successful pathogen identification will support accurate treatment.

Keywords: Coronavirus disease-2019 (COVID-19); non-COVID-19; pathogens; differential diagnosis

Submitted Sep 24, 2020. Accepted for publication Jan 29, 2021.

doi: $10.21037 /$ atm-20-6600

View this article at: http://dx.doi.org/10.21037/atm-20-6600

\section{Introduction}

Coronavirus disease-2019 (COVID-19) has crossed borders rapidly around the world since its outbreak in December 2019, leading to more than 70 million cases in over 160 countries up to 14 December 2020 (1). Before the severe acute respiratory syndrome (SARS) outbreak due to severe acute respiratory syndrome coronavirus-1 (SARS-CoV-1) in 2003 (2), coronaviruses were not considered a serious 
medical concern. However, another fatal coronavirus named Middle East respiratory syndrome coronavirus (MERS$\mathrm{CoV}$ ) emerged and spread to several countries in 2013 (3), which highlighted coronavirus infection as a potential threat to human health. Sequence homology analysis showed that SARS-CoV-2 appears to be associated with bat-derived SARS-like coronaviruses (bat-SL-CoVZC45 and bat-SLCoVZXC21) (with $88 \%$ identity), however, it is different from MERS-CoV (approximately 50\%) and SARS-CoV-1 (approximately 79\%) (4). However, several studies have proven that SARS-CoV-2 has high rates of human-tohuman transmission $(5,6)$ and morbidity $(7)$, resulting in a global pandemic (8).

Although SARS-CoV-2 deserves our attention because of its high transmissibility and lethality, we cannot ignore infections with other pathogens. There have been many reports ofCOVID-19 patients with mixed pathogen infections, including coinfections with bacteria and fungi $(7,9)$ and coinfections with other respiratory viruses $(10)$. To complicate matters, the epidemiology of SARS-CoV-2 are very similar to that of other respiratory viruses, including influenza $(11,12)$ and rhinoviruses $(13)$, and with that of bacterial infections such as mycoplasma (14). Several reports of COVID-19 have indicated that not all admitted patients were infected with SARS-CoV-2, highlighting the urgent need to determine the causes of fever and febrile illness to support the selection of more appropriate and effective treatment (15). Achieving an accurate differential diagnosis will help reduce the consumption of clinical resources while also improving the prevention and control of the pandemic.

However, to the best of our knowledge, there are few reports regarding the comparison of clinical features and treatment between COVID-19 patients and those with infectious pneumonia caused by other pathogens. Furthermore, previous studies did not distinguish COVID-19 cases from other febrile patients with clinical examinations. Therefore, this study was designed to retrospectively review and analyze the pathogen spectrum, clinical characteristics, laboratory parameters, and treatment of all suspected COVID-19 patients recruited in the isolation ward of our hospital. By comparing confirmed COVID-19 and non-COVID-19 patients, we provide new insight into the differential diagnosis and treatment of suspected COVID-19 patients during the ongoing pandemic. This study distinguishes COVID-19 cases from other febrile patients with clinical examinations.

We present the following article in accordance with the STROBE reporting checklist (available at http://dx.doi. org/10.21037/atm-20-6600).

\section{Methods}

\section{Study design and patients}

Suspected COVID-19 patients were recruited from January 23 to May 23, 2020, for this single-center, retrospective study at the First Affiliated Hospital of Guangzhou Medical University (Guangzhou), China, one of the hospitals designated for COVID-19 patients. According to the provisions employed by the Guangdong government, all suspected and confirmed patients were received and treated without any selection criteria. We set up a ward to isolate and treat all suspected and confirmed patients and shared all data from confirmed cases with the Center for Disease Control and Prevention (CDC), Guangdong.

The suspected COVID-19 cases were defined according to the interim guidance of the World Health Organization (WHO) and China $(16,17)$. The guidance states that the COVID-19 diagnosis is made based on a combination of a comprehensive epidemiological history and clinical manifestations. The factors related to the epidemiological history were: (I) residence or history of travel to Hubei Province or the surrounding areas or other case-reporting places within two weeks before the onset of disease; (II) contact with a confirmed COVID-19 patient being confirmed via reverse transcription-polymerase chain reaction within two weeks before the onset of the disease; (III) contact with a person reporting respiratory symptoms or fever, who came from Hubei Province or the surrounding areas or case-reporting communities within two weeks before the onset of the disease; and (IV) association with a disease cluster ( $\geq 2$ cases of respiratory symptoms and fever in a small working area e.g., home, office, or school class within two-weeks). The clinical manifestations were as follows: (I) respiratory symptoms and fever; (II) COVID-19 imaging features characteristic; and (III) normal or decreased total leukocyte count and normal or decreased lymphocyte count in the initial stages of the disease. Patients were defined as suspected COVID-19 patients if they demonstrated any one of the epidemiological-history criteria and any two of the clinical manifestation criteria. However, patients with unclear epidemiological-history demonstrating three of the clinical manifestations were also considered potential COVID-19 patients. The study conformed to the provisions of the Declaration of Helsinki (as revised in 2013). The ethics 
committee of the First Affiliated Hospital of Guangzhou Medical University approved this study (Ethical number: 2020-92). All participants have obtained informed consent before participating.

\section{Procedures and data collection}

The clinical charts, laboratory findings, nursing records, and chest X-rays of all suspected COVID-19 patients were reviewed in the isolation ward of our hospital. Epidemiological, laboratory, clinical, radiological characteristics, treatment, and outcome data were obtained from the electronic medical records through the standardized data collection forms (modified caserecord forms for severe acute respiratory infection clinical characterization, released by the International Severe Acute Respiratory and Emerging Infection Consortium). The follow up of clinical outcomes was made till $23^{\text {rd }}$ May 2020. Data were obtained via direct communication with the attending physicians and other healthcare providers if clarification was needed or data were missing from the records. The data collection forms were independently reviewed by two researchers (ZTL and SQL) to double the collected data for validity.

\section{Pathogen detection}

Pathogens were detected by traditional culture/ histopathology, serum antibody tests, and nucleic acid amplification tests, including RT-PCR, for bacteria/ fungi, mycoplasma, and viruses, respectively. Clinical metagenomics next-generation sequencing (mNGS) was used when the pathogen was difficult to identify. Testing was performed either in our hospital or by the companies recognized by the government as having a testing qualification. Finally, the pathogenic agents identified in suspected COVID-19 patients were determined by combining the clinical manifestations and pathogen test results.

\section{Statistical analysis}

When the measurement data were normally distributed, mean values were compared using independent group $t$-tests. Otherwise, the Mann-Whitney test was used. With regards to nonnormally distributed data, the measurement data were presented as the medians and inter quartile ranges (IQRs). Count data were expressed in terms of frequencies and percentages. Comparisons between groups were performed using $t$-tests, while Fisher's exact test was applied for limited data. Statistical analysis was performed through SPSS Software (v25.0; IBM, Armonk, NY, USA). A P value of less than 0.05 was considered significant statistically.

\section{Results}

From January 23 to May 23, 2020, approximately 1,875 patients with fever attended the fever clinic of our hospital. Upon assessment by the receiving doctor, 134 patients were identified as suspected COVID-19 patients and were admitted to the isolation ward of the designated hospital. These 134 patients were enrolled in this study, including 23 COVID-19 patients confirmed with laboratory testing (mainly RT-PCR positive) and 111 non-COVID-19 patients (RT-PCR negative) (Figure 1).

Among the 134 suspected COVID-19 patients, the median age was 48 years old (IQR, 32-59); 74 (55.2\%) were aged 15-49 years, and $35(26.1 \%)$ were aged 50-64 years. $58(43.2 \%)$ were male. In terms of smoking history, 106 (79.1\%) had never smoked, 16 (11.9\%) had quit, and 12 (7.5\%) were still smoking. Among the 134 patients, 19 (7.4\%) had hypertension, 14 (10.4\%) had diabetes, 7 (5.2\%) had coronary heart disease, 3 (2.2\%) had cerebrovascular disease, 2 (3.2\%) had chronic obstructive pulmonary disease, and $5(3.7 \%)$ had cancer. Twenty (14.9\%) had been to Hubei Province. Comparing the non-COVID-19 and COVID-19 groups, no significant difference was observed at baseline, except for the history of travel to Hubei Province, which was more common in the COVID-19 group (60.9\% vs. 5.4\%, $\mathrm{P}<0.05)$ (Table 1$)$.

The primary task of clinical diagnosis and treatment is to identify the infectious agent. Thus, multiple detection methods, including traditional culture methods, serum antibodies, histopathology, and nucleic acid amplification tests, are used to identify the pathogen. However, in our study, only 84 (62.7\%) of the 134 pneumonia patients had an easily identifiable pathogen, and the remaining 50 (37.3\%) patients were infected with unknown pathogens (Figure 2A). In some cases, the infectious agent could be identified with one detection method, while others needed more than one detection method (Figure 2B). The final determination of pathogenic agents must be made by combining the detection results and clinical manifestations. Overall, $53(63.1 \%)$ of the 84 subjects presented with viral infections, followed by10 (11.9\%) cases of bacterial infections, 14 (16.7\%) cases of mycoplasma infections, and 


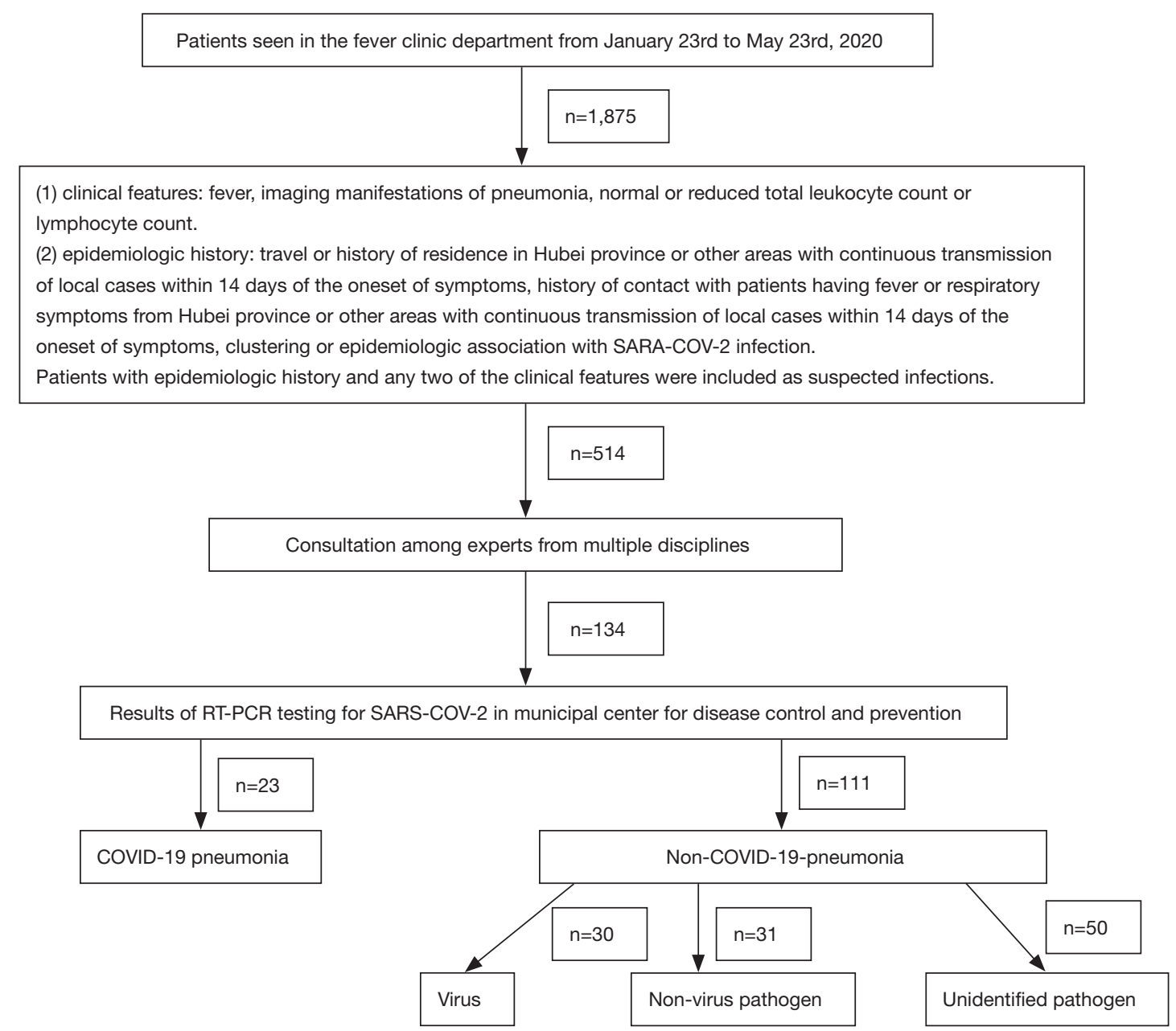

Figure 1 Flowchart for the enrollment of patients in this study. SARS-CoV-2, severe acute respiratory syndrome coronavirus 2; RT-PCR, reverse transcription-polymerase chain reaction; COVID-19, coronavirus disease 2019.

$5(6.0 \%)$ cases of tuberculosis. A further 3 cases $(3.3 \%)$ of mixed viral infections were identified, and there was one case each of fungal infection, unidentified bacterial-viral coinfection, and chlamydia-viral coinfection (Figure 2C). In Figure 2D,E, the classifications of viruses and bacteria identified in two-thirds of the study population are shown. There were 53 viruses in 84 patients, among which 23 were SARS-CoV-2, and the remaining were common viruses, i.e., influenza-A virus, rhinovirus, parainfluenza virus, adenovirus, and herpes virus (Figure $2 D$ ). There were also 31 species of bacteria and other pathogens, the most common being mycoplasma, tuberculosis, Haemophilus parainfluenzae, and Pseudomonas aeruginosa (Figure 2E).

Understanding the differences between COVID-19 and other diseases is vital for combatting the ongoing pandemic. The 84 patients with clearly identified infectious agents (excluding the three patients with mixed infections) were divided into three groups: group A (COVID-19; 23 patients), group $\mathrm{B}$ (other viral infections; 30 patients), and group C (nonviral infections; 31 patients). The clinical features, radiological characteristics, and treatments were compared among these groups. As shown in Table 2, 77(91.7\%) of the 84 patients had a fever during hospitalization, and 25 (29.8\%) had a peak temperature $>38^{\circ} \mathrm{C}$. More than half of the COVID-19 patients (52.2\%) had high fevers $>38^{\circ} \mathrm{C}$, which were higher than the peak temperatures in groups B and C. The other symptoms, including fatigue $34.8 \%$ vs. $23.3 \%$ vs. $9.6 \%$ in groups $\mathrm{A}$, $\mathrm{B}$, and C, respectively) and muscle aches (39.1\% vs. $10.0 \%$ vs. $9.6 \%$ in groups $\mathrm{A}, \mathrm{B}$, and $\mathrm{C}$, respectively), were more commonly seen in patients with viral infections, especially in the COVID-19 group. Laboratory examinations of 
Table 1 Baseline characteristics of study population

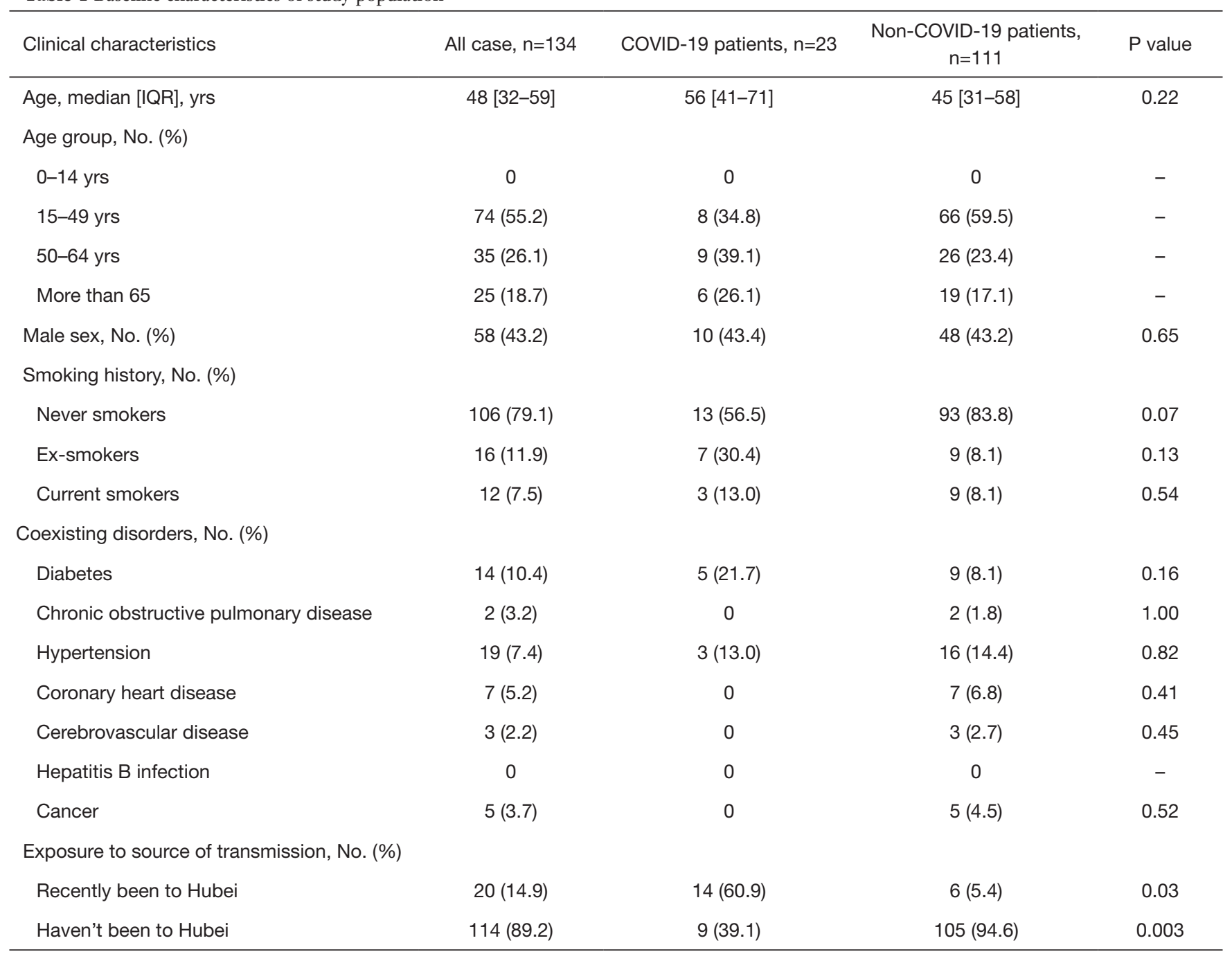

COVID-19, coronavirus disease 2019.

these patients showed that the lymphocyte count of more than half of theCOVID-19 patients (52.2\%) was less than $0.8 \times 10^{-9} / \mathrm{L}$, which was significantly different from group $\mathrm{C}(\mathrm{P}<0.05)$. We also found that the lactate dehydrogenase (LDH) levels and median neutrophil-tolymphocyte ratio (NLR) (IQR) were significantly higher in COVID-19 patients than those in groups $\mathrm{B}$ and $\mathrm{C}(\mathrm{P}<0.05)$. Furthermore, the radiological characteristics were also compared among the three groups. In COVID-19 patients, the infection foci often involved both lungs $(95.7 \% \mathrm{vs}$. $56.7 \%$ vs. $48.4 \%$ among groups A, B, and C, respectively, $\mathrm{P}<0.05$ ), with ground-glass opacities (GGOs; Figure 3 and Table S1).

The accurate detection of pathogens and comparison of clinical characteristics ultimately direct the clinical treatment and improve the treatment rate. Following the identification of a known pathogen, all COVID-19 patients received antiviral and traditional Chinese medicine treatment, while $76.7 \%$ of patients in group B received antivirals. However, only $12.9 \%$ of patients in group $\mathrm{C}$ received antiviral treatment $(\mathrm{P}<0.05)$. Furthermore, only $13.3 \%$ of patients in group B but $61.3 \%$ of patients in group $\mathrm{C}$ received antibiotics $(\mathrm{P}<0.05)$. Additionally, oxygen therapy was a common treatment in this cohort of COVID-19 patients (47.8\%), but nearly half (43.5\%) COVID-19 patients required mechanical ventilation. Precise differential diagnosis and treatment led to a low death rate and significantly reduced duration of hospital stay 
A

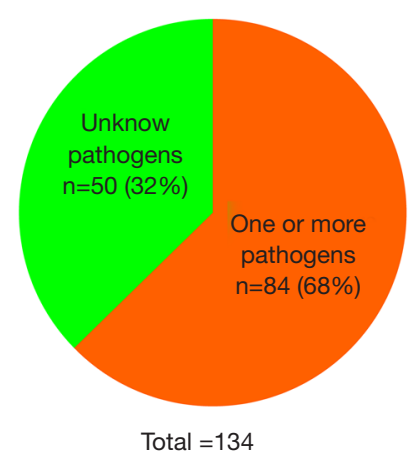

B

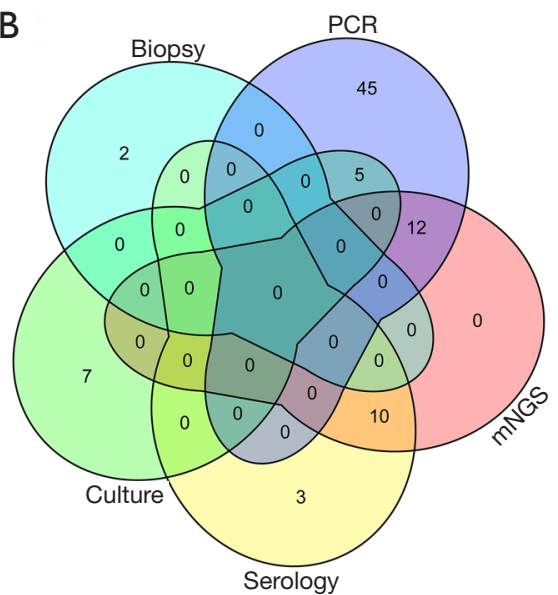

C

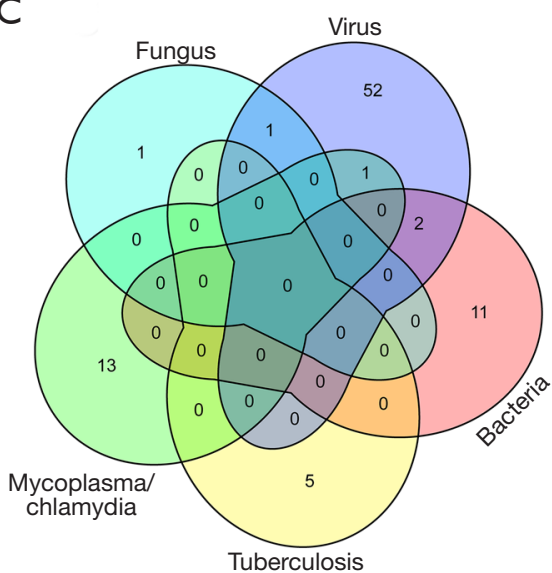

D

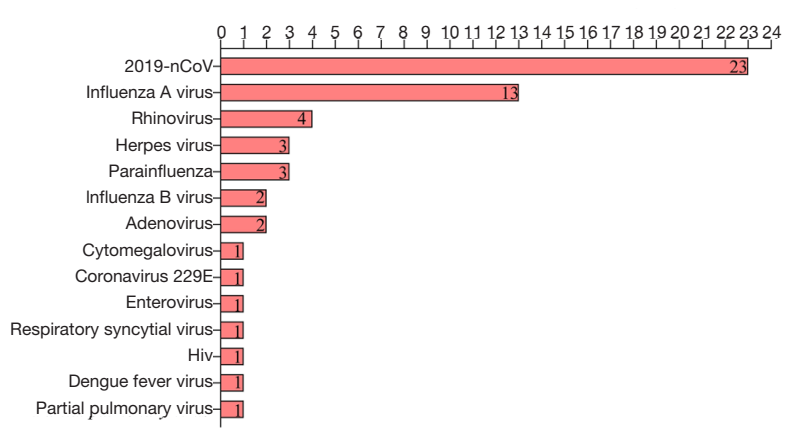

E

Species of bacteria and other pathogens $(n=3)$

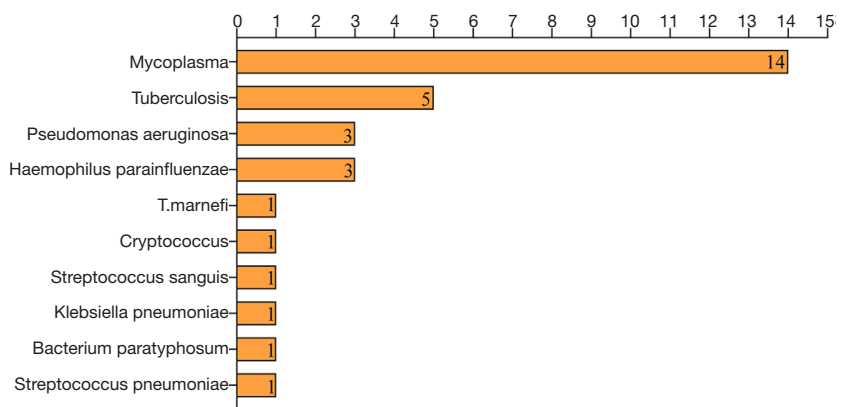

Figure 2 Detection of pathogens in suspected COVID-19 patients. (A) Percentage of identified and unidentified pathogens. (B,C) Detection methods used in our study. (D,E) Show the pathogen spectrum. COVID-19, coronavirus disease 2019.

in group $\mathrm{B}$ and $\mathrm{C}$ patients compared with patients in group A $(29 \pm 5.8$ vs. $4.7 \pm 3.3$ vs. $6.3 \pm 3.9$ days, groups A, B, and C, respectively; $\mathrm{P}<0.001)$. Furthermore, hospitalization costs were also significantly lower in non-COVID-19 patients

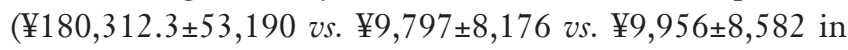
groups $\mathrm{A}, \mathrm{B}$, and $\mathrm{C}$, respectively; $\mathrm{P}<0.001$ ) (Table 3).

\section{Discussion}

In this study, we describe respiratory infections with other pathogens during the COVID-19 epidemic in China. Our findings indicate that for those suspected COVID-19 patients presenting with fever or respiratory symptoms, in addition to SARS-CoV-2, other pathogens, including seasonal respiratory viruses and bacteria, should also be considered. Furthermore, epidemiological history; clinical symptoms of high fever, fatigue, and muscle ache; laboratory findings of low lymphocyte counts, elevated IQRs, and elevated LDH levels; and radiological characteristics of GGOs involving both lungs help differentiate COVID-19 from other viral or bacterial infections. Finally, clear identification of the pathogen and differential diagnosis of the disease contribute to the administration of the appropriate antiviral and antimicrobial treatment, thus improving the treatment rate, reducing hospital stay durations, and saving medical resources.

Respiratory infectious disease is the world's fourth leading cause of death, with all infectious diseases ranking first, accounting for $30.21 \%(18-20)$. Additionally, the emergence and re-emergence of respiratory tract pathogens are continuous (21), including the previously unknown SARS-CoV-2, which has now caused a global pandemic. The rapid and accurate detection of pathogens, drug resistance, and mutations, as well as the identification of unknown pathogens, can be difficult, thus causing problems with regard to clinical diagnosis and treatment. 
Table 2 Comparison of clinical characteristics of different pathogens

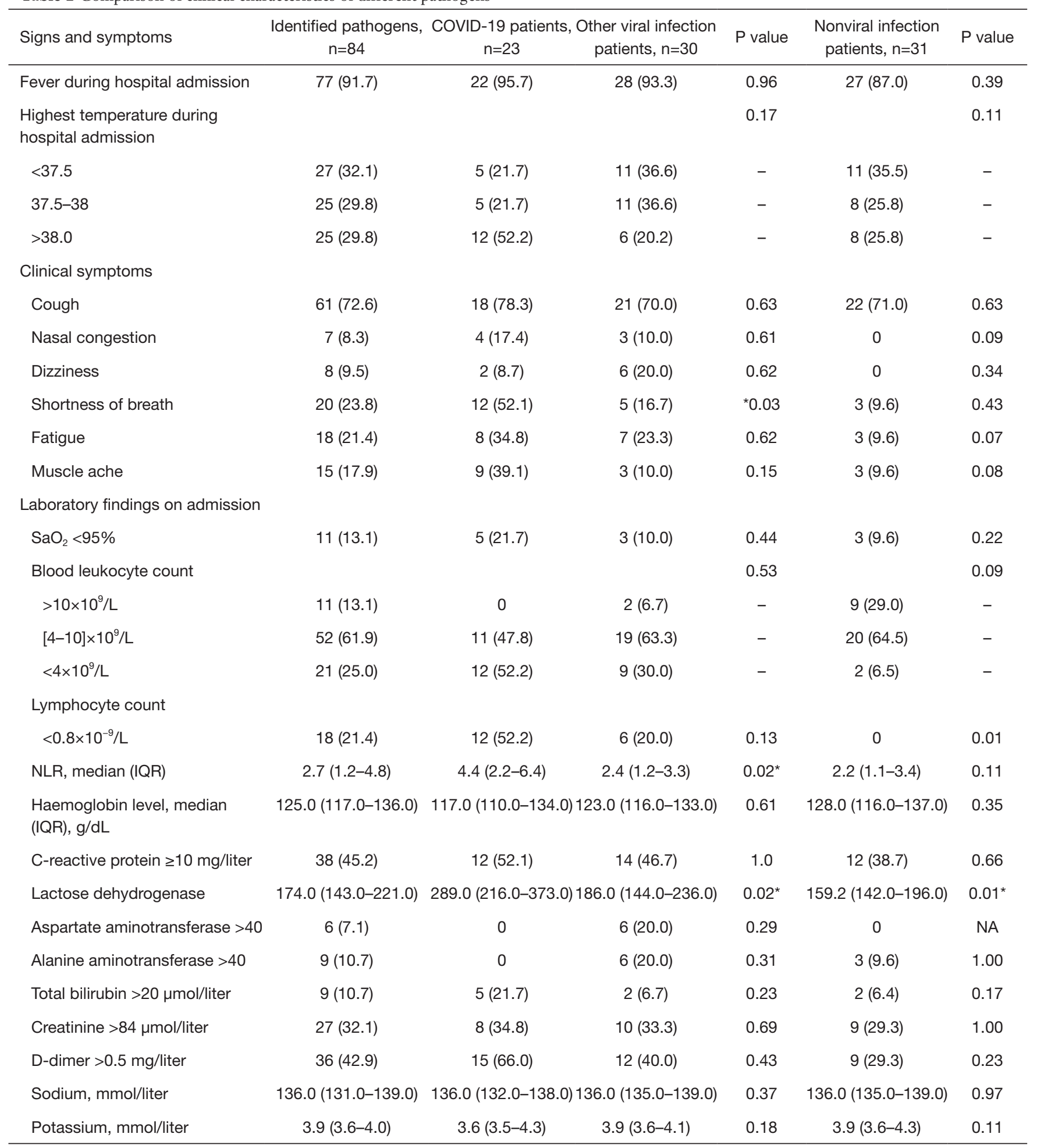

*, P value less than 0.05. COVID-19, coronavirus disease 2019; NLR, neutrophil-to-lymphocyte ratio. 


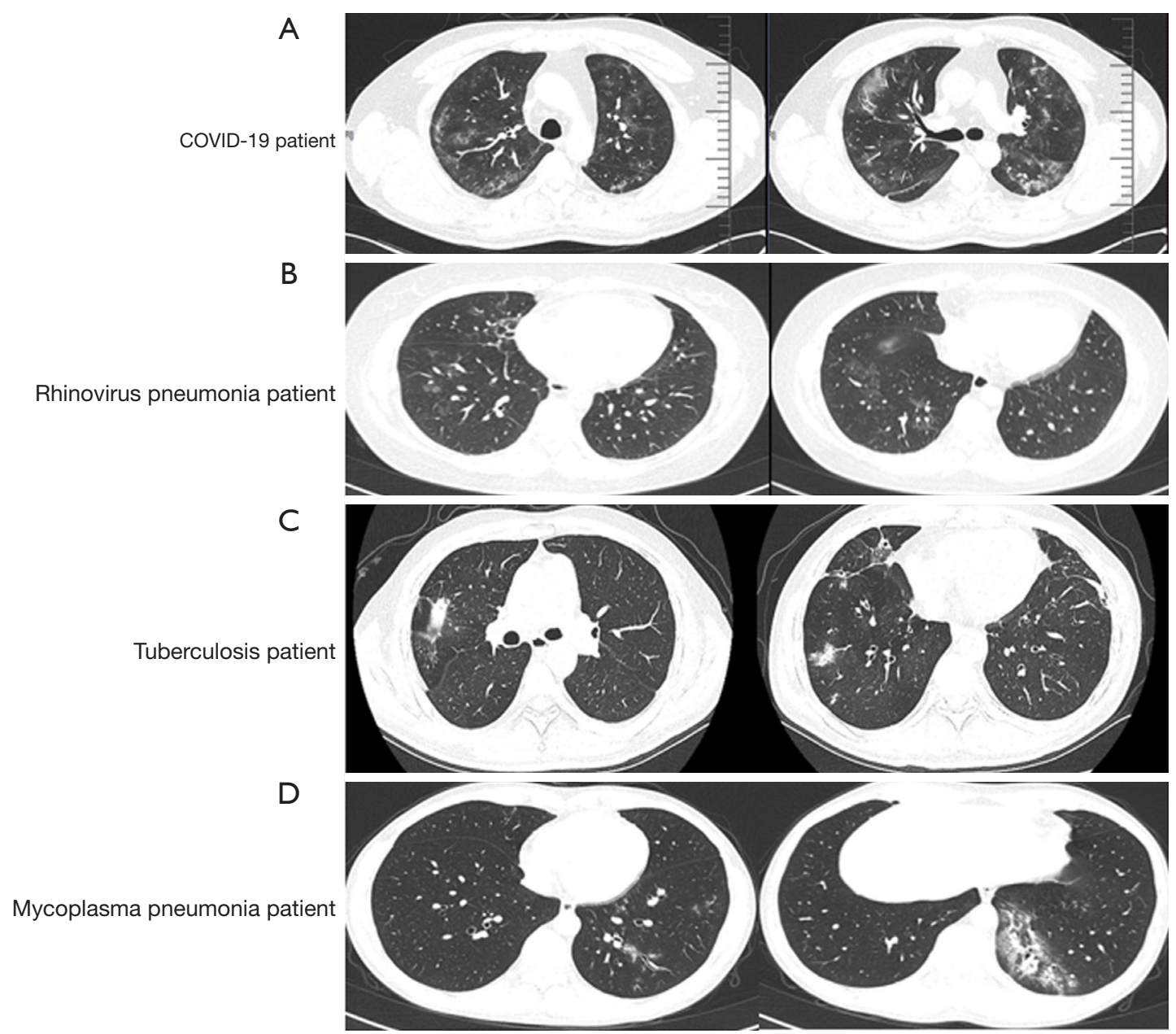

Figure 3 Characteristic chest CT (lung window) in suspected COVID-19 patients. (A) Patient with confirmed COVID-19. Multiple ground-glass opacity involvement in several lobes in both lungs, with most of the exudation distributed under the pleura. Some lesions are fused, and consolidation is visible. (B) Patient with ground-glass changes in the double lower medial pulmonary bands, finally diagnosed with rhinovirus pneumonia. (C) Patient with multiple nodules in the right lung with ground-glass changes. Final diagnosis of tuberculosis. (D) Patient with ground-glass opacities combined with consolidation in the lower left lung, finally diagnosed with Mycoplasma pneumoniae infection. COVID-19, coronavirus disease 2019.

A rapid and accurate diagnosis ensures treatment with the appropriate antibiotics or antivirals and improves patient outcomes. Similarly, the rapid identification of causative agents is important for facilitating rapid responses during outbreak investigations. Real-time PCR is used as a diagnostic tool using a nasal swab, tracheal aspirate, or bronchoalveolar lavage samples. Computed tomography findings are important for both diagnosis and follow-up. To date, there is no evidence of any effective treatment for COVID-19. The main therapies being used to treat the disease are antiviral drugs and respiratory therapy and mechanical ventilators $(22,23)$. Furthermore, numerous vaccines are underway for COVID-19 (24). However, the existing pathogen diagnosis technology is still unable to meet these needs. In our study, common detection methods, including traditional culture methods, serum antibody detection, histopathology, and nucleic acid amplification tests (RT-PCR and mNGS), were used. Regrettably, we found that approximately $37.3 \%$ of cases were caused by unidentifiable pathogens (Figures 1,2). Previous studies have also shown that up to $60 \%$ of cases are still treated with antibiotics without the etiology being known (25-27). This issue arises from defects in the existing detection methods. The traditional detection method of 


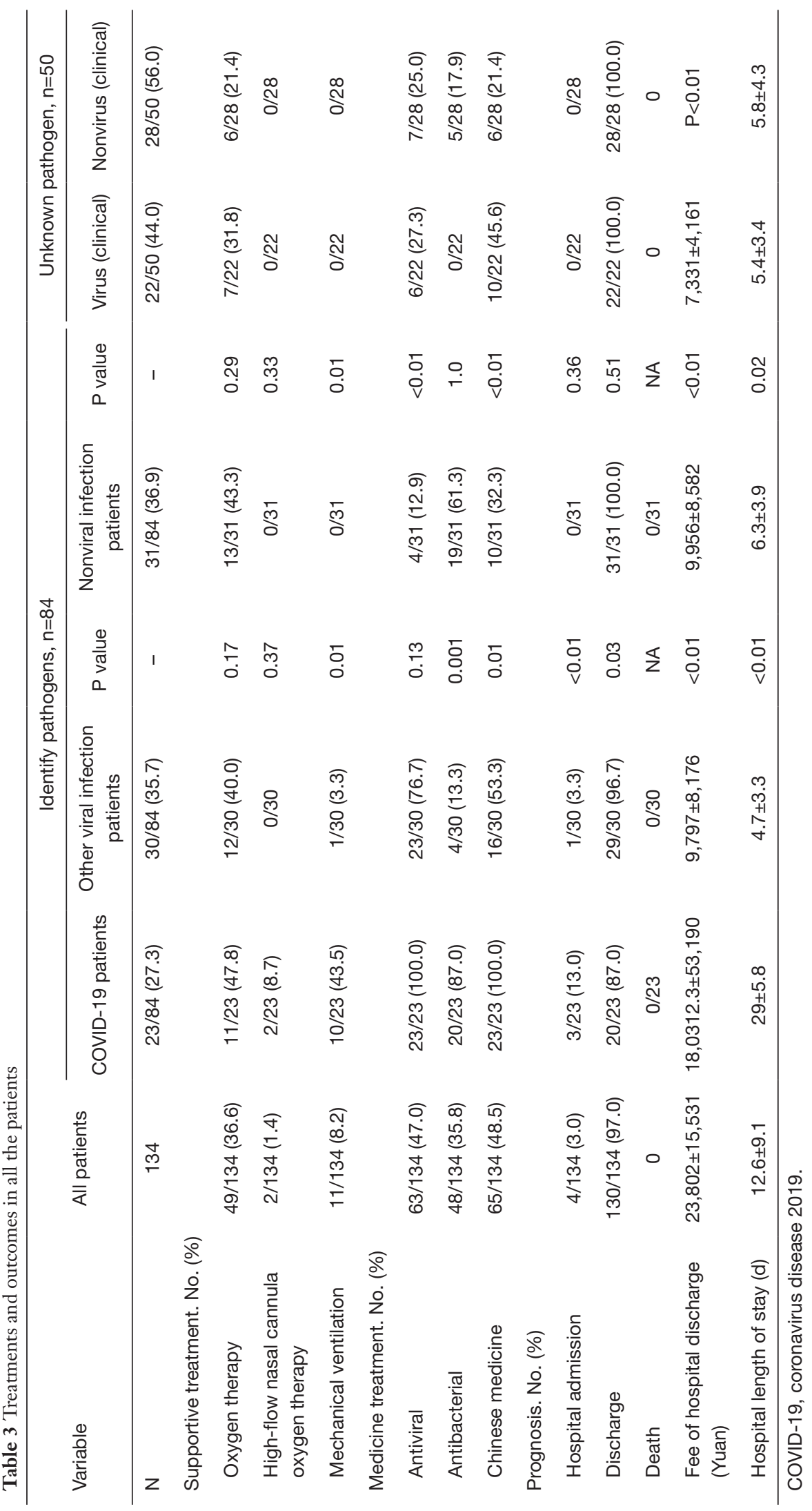


pathogen culture has been in use for 70 years (28). However, it is of limited use as a guide for appropriate clinical management of acute infections, mainly due to slow sampleto-result turnaround (29). Though nucleic acid amplification tests (e.g., RT-PCR) are highly specific, rapid, and sensitive, there remain some limitations on multiplexing, thus need to update PCR-based methods urgently to include emerging resistance mutations and genes (30,31). Furthermore, nextgeneration sequencing platforms, an emerging detection method, are frequently employed for metagenomics sequencing, however, they are rendered unsuitable for rapid detection due to its requirement of the sequencing run to be completed before analysis can begin (32). Therefore, new and flexible detection methods need to be developed to facilitate efficient clinical diagnosis and treatment.

Additionally, different diseases often present with similar symptoms. According to previous studies on the clinical characteristics of COVID-19 (5,6,33), fever, cough, and fatigue are the most common clinical manifestations in patients with COVID-19. However, in our study, we found that these symptoms were not only presented by COVID-19 patients but also presented by patients infected with other viruses and mycoplasma (Table 2). This finding suggests that COVID-19 patients cannot be distinguished from other patients merely by clinical symptoms, and the identification of pathogens should not be limited to SARS-CoV-2 during the epidemic of COVID-19. The SARS-CoV-2 outbreak occurred during the seasonal epidemic periods of influenza and rhinoviruses $(13,34)$ and the post epidemic period of mycoplasma infections (14). Several studies have found that for influenza virus infection, rhinovirus infection has played an important role in the etiology of annual nosocomial outbreaks in recent years $(12,13,35)$. In our study, confirmed COVID-19 accounted for only approximately $17.2 \%$ of the 134 suspected COVID-19 cases, with other pathogens, such as influenza, being responsible for the main proportion (Figure 2). Because of this overlap in symptoms, several previous studies have suggested that chest CT helps screen suspected cases of SARS-CoV-2 infection $(36,37)$. While there are some typical characteristics in COVID-19 patients, such as GGOs involving both lungs (Figure 3 and Table S1), we realized that the chest CTs of COVID-19 patients were somewhat similar to those of patients with other viral infections (38). Furthermore, we also found that some patients with leukemia or solid organ tumors with a history of chemotherapy but no epidemiological history may also have GGOs on their chest CT with fever and normal or low white blood cell counts. One such patient was identified as a suspected COVID-19 case and admitted to our ward. This patient was finally diagnosed with immunosuppressive host pneumonia, which was identified as pneumocystis pneumonia with a concomitant viral infection. Therefore, the combination of many factors should be considered when differentiating COVID-19 from infections with other pathogens, especially in suspected patients with false-negative RT-PCR results (39). Many factors are important in making a diagnosis of COVID-19, including epidemiological history (Table 1), because most COVID-19 patients have epidemiological differences compared to other patients (7); symptoms, such as fever, fatigue, and muscle aches (Table 2); and laboratory findings, such as absolute counts of lymphocytes, which in most COVID-19 patients are significantly reduced compared with those in patients with other respiratory illnesses $(\mathrm{P}<0.05)$, with $52.2 \%$ of patients demonstrating counts less than $0.8 \times 10^{-9} / \mathrm{L}$. Besides, LDH levels are significantly higher in COVID-19 patients than in non-COVID-19 patients (Table 2). Laboratory detection indices were also shown to have some diagnostic value for COVID-19 in other studies (7); thus, combining these with chest CT will greatly improve the differential diagnosis of COVID-19.

Identification of the pathogen and the differential diagnosis of COVID-19 are essential for implementing appropriate and effective clinical treatment. While other studies have shown that up to $60 \%$ of cases are treated with antibiotics despite being of unknown etiology (25-27), we administered appropriate antiviral and antibacterial treatments in our isolation ward for suspected COVID-19 patients (Table 3), which was made possible by the high detection rate of pathogens $(62.7 \%)$. The use of a variety of detection methods enabled us to effectively identify the responsible pathogens, thereby allowing us to provide precise treatment as often as possible. For the $37.3 \%$ of patients with unclear etiological diagnoses, we made clinical judgments based on the relevant laboratory examinations and chest CT and created a tailored treatment plan for every patient. By observing the clinical efficacy of the treatments and tracking patient prognosis, we found that there were no patients who deteriorated because of delayed antibiotic use. Our application rate of antibiotics $(35.8 \%)$ was relatively low, and the average hospitalization time for non-COVID-19 patients was much shorter than that for COVID-19 patients, with a lower average cost. This situation helps reduce the pressure on medical staff and mitigate social panic during the outbreak. Governments will not be able to minimize both deaths from coronavirus 
disease 2019 (COVID-19) and the economic impact of viral spread. Keeping mortality as low as possible will be the highest priority for individuals; hence governments must put in place measures to ameliorate the inevitable economic downturn (40).

Of course, there were some limitations to the present study. First, the number of COVID-19 cases was small because this was a single-center study. Second, all patients' information was collected in the isolation ward, where patients were treated during the early stage of the disease, and some patients were transferred to other departments or hospitals when the pathogen was identified. Third, most confirmed patients had not been discharged at the time of the writing of this report. Consequently, we were unable to estimate either the predictors of mortality or the case-fatality rate. Finally, the current did not follow all the statistical tools of retrospective research. This may increase the deviation of research results. Hence, a more comprehensive statistical analysis with large sample data is required in future studies.

\section{Conclusions}

Not all patients presenting with fever or a relevant epidemiological history were infected with SARS-CoV-2. Pathogens such as other viruses and bacteria, including mycoplasma, should be considered when making a diagnosis of SARS-CoV-2 infection. Therefore, it is important to improve the relevant tests and make a clear and rapid diagnosis to facilitate the administration of appropriate treatment, mitigate panic, and provide the best outcome for patients.

\section{Acknowledgments}

The authors thank the patients; nurses and clinical staff for providing care to the patients; staff at the respiratory medicine department of the hospital; staff at the clinical laboratory; technical staff at the Department of State Key Laboratory of Respiratory Disease for their excellent assistance; and staff at Guangdong CDC for the diagnosis of SARS-CoV-2. The authors also like to thank Mingzhou Xie for helping in beautifying some illustration in this manuscript. The authors also like to thank AJE for editing the language of the text in this manuscript.

Funding: This work was funded by the Scientific and Technological Emergency Special of Guangdong Province for Prevention and Controlling of COVID-19 (Grant number: 2020B1111340004); the Guangzhou Institute of Respiratory Health Open Project (Funds provided by China Evergrande Group) (Project No. 2020GIRHHMS14); the National Natural Science Foundation of China (Grant number: 62041302) and the Innovative and Strategic Program of Guangdong's Scientific and Technological Policy (Grant number: 2020B111126005); the Traditional Chinese Medicine Bureau of Guangdong Province (Grant number: 2020ZYYJ05); Penghua Care Fund to the Medical Pioneers against Covid-19 of Shenzhen Social Commonweal Foundation; Guangzhou Science and Technology Innovation Development Special Fund (Grant number: 202008010002); and by the Guangzhou Institute of Respiratory Health.

\section{Footnote}

Reporting Checklist: The authors have completed the STROBE reporting checklist. Available at http://dx.doi. org/10.21037/atm-20-6600

Data Sharing Statement: Available at http://dx.doi. org/10.21037/atm-20-6600

Peer Review File: Available at http://dx.doi.org/10.21037/ atm-20-6600

Conflicts of Interest: All authors have completed the ICMJE uniform disclosure form (available at http://dx.doi. org/10.21037/atm-20-6600). NZ serves as an unpaid honorary editor-in-chief of Annals of Translational Medicine from Jan 2019 to Dec 2024. The other authors have no conflicts of interest to declare.

Ethics Statement: The authors are accountable for all aspects of the work in ensuring that questions related to the accuracy or integrity of any part of the work are appropriately investigated and resolved. The study conformed to the provisions of the Declaration of Helsinki (as revised in 2013). The ethics committee of the First Affiliated Hospital of Guangzhou Medical University approved this study (Ethical number: 2020-92). All participants have obtained informed consent before participating.

Open Access Statement: This is an Open Access article distributed in accordance with the Creative Commons Attribution-NonCommercial-NoDerivs 4.0 International 


\section{Page 12 of 13}

License (CC BY-NC-ND 4.0), which permits the noncommercial replication and distribution of the article with the strict proviso that no changes or edits are made and the original work is properly cited (including links to both the formal publication through the relevant DOI and the license). See: https://creativecommons.org/licenses/by-nc-nd/4.0/.

\section{References}

1. Organization, W.H., Coronavirus disease (COVID-19) Weekly Epidemiological Update and Weekly Operational Update. [cited 2020 Dec 14]; Available online: https://www.who.int/publications/m/item/weeklyepidemiological-update. 14-december-2020.

2. Ksiazek TG, Erdman D, Goldsmith CS, et al. A novel coronavirus associated with severe acute respiratory syndrome. New Eng J Med 2003;348:1953-66.

3. Zaki AM, van Boheemen S, Bestebroer TM, et al. Isolation of a novel coronavirus from a man with pneumonia in Saudi Arabia. N Engl J Med 2012;367:1814-20.

4. Guan WJ, Ni ZY, Hu Y, et al, Clinical characteristics of coronavirus disease 2019 in China. N Engl J Med 2020;382:1708-20.

5. Huang C, Wang Y, Li X, et al. Clinical features of patients infected with 2019 novel coronavirus in Wuhan, China. Lancet 2020;395:497-506.

6. Wang D, Hu B, Hu C, et al. Clinical Characteristics of 138 Hospitalized Patients With 2019 Novel CoronavirusInfected Pneumonia in Wuhan, China. JAMA 2020;323:1061-9.

7. Zhao D, Yao F, Wang L, et al. A comparative study on the clinical features of COVID-19 pneumonia to other pneumonias. Clin Infect Dis 2020;71:756-761.

8. Phelan AL, Katz R, Gostin LO. The Novel Coronavirus Originating in Wuhan, China: Challenges for Global Health Governance. JAMA 2020;323:709-10.

9. Yang J, Zheng Y, Gou X, et al. Prevalence of comorbidities and its effects in patients infected with SARS-CoV-2: a systematic review and meta-analysis. Int J Infect Dis 2020;94:91-5.

10. Wu X, Cai Y, Huang X, et al. Co-infection with SARSCoV-2 and Influenza A Virus in Patient with Pneumonia, China. Emerg Infect Dis 2020;26:1324-6.

11. Chinese National Influenza Center [http://www.chinaivdc. $\mathrm{cn} / \mathrm{cnic} /$, China's weekly influenza surveillance report [cited 2020 Dec 18]. Available online: http://www. chinaivdc.cn/cnic/

12. Azziz Baumgartner E, Dao CN, Nasreen S, et al.

\section{Li et al. Clinical characteristics of confirmed COVID-19 cases}

Seasonality, timing, and climate drivers of influenza activity worldwide. J Infect Dis 2012;206:838-46.

13. To KKW, Yip CCY, Yuen KY. Rhinovirus - From bench to bedside. J Formos Med Assoc 2017;116:496-504.

14. Qu J, Yang C, Bao F, et al. Epidemiological characterization of respiratory tract infections caused by Mycoplasma pneumoniae during epidemic and postepidemic periods in North China, from 2011 to 2016. BMC Infect Dis 2018;18:335.

15. Guan WJ, Chen R, Zhong N. Strategies for the prevention and management of coronavirus disease 2019. Eur Respir J 2020;55:2000597.

16. Chinese Center for Disease Control and Prevention [Internet]. [cited 2020 March 03]. Office of State Administration of traditional Chinese Medicine; [about 1 screens]. Available online: http://www.chinacdc. cn/jkzt/crb/zl/szkb_11803/jszl_11815/202003/ t20200305_214142.html

17. World Healthy Organization [Internet]. [cited $2020 \mathrm{Feb} 8$ ]. [about 2 screens]. Available online: https://www.who.int/ publications-detail/clinical-management-ofsevere-acuterespiratory-infection-when-novel-coronavirus-(ncov)infection-is-suspected

18. World Healthy Organization [Internet]. [update 2004]. Media Center; [about 2 screens]. Available online: https:// www.who.int/healthinfo/global_burden_disease/2004_ report_update/en

19. GBD 2016 Lower Respiratory Infections Collaborators. Estimates of the global, regional, and national morbidity, mortality, and aetiologies of lower respiratory infections in 195 countries, 1990-2016: a systematic analysis for the Global Burden of Disease Study 2016. Lancet Infect Dis 2018;18:1191-210.

20. Murdoch DR, Howie SRC. The global burden of lower respiratory infections: making progress, but we need to do better. Lancet Infect Dis 2018;18:1162-3.

21. Gao GF. From "A"IV to "Z"IKV: Attacks from Emerging and Re-emerging Pathogens. Cell 2018;172:1157-9.

22. Pascarella G, Strumia A, Piliego C, et al. COVID-19 diagnosis and management: a comprehensive review. J Intern Med 2020;288:192-206.

23. Wiersinga WJ, Rhodes A, Cheng AC, et al. Pathophysiology, Transmission, Diagnosis, and Treatment of Coronavirus Disease 2019 (COVID-19): A Review. JAMA 2020;324:782-93.

24. Raja AT, Alshamsan A, Al-Jedai A. Current COVID-19 vaccine candidates: Implications in the Saudi population. Saudi Pharm J 2020;28:1743-8. 
25. Schlaberg R, Chiu CY, Miller S, et al. Validation of Metagenomic Next-Generation Sequencing Tests for Universal Pathogen Detection. Arch Pathol Lab Med 2017;141:776-86.

26. Ewig S, Torres A, Angeles Marcos M, et al. Factors associated with unknown aetiology in patients with community-acquired pneumonia. Eur Respir J 2002;20:1254-62.

27. van Gageldonk-Lafeber AB, Heijnen ML, Bartelds AI, et al. A Case-Control Study of Acute Respiratory Tract Infection in General Practice Patients in The Netherlands. Clin Infect Dis 2005;41:490-7.

28. McINTOSH J. Emergency Pathology Service. Lancet 1946;1:669.

29. Charalampous T, Kay GL, Richardson H, et al. Nanopore metagenomics enables rapid clinical diagnosis of bacterial lower respiratory infection. Nat Biotechnol 2019;37:783-92.

30. Hassibi A, Manickam A, Singh R, et al. Multiplexed identification, quantification and genotyping of infectious agents using a semiconductor biochip. Nat Biotechnol 2018;36:738-45.

31. Buchan BW, Ledeboer NA. Emerging technologies for the clinical microbiology laboratory. Clin Microbiol Rev 2014;27:783-822.

32. Tausch SH, Benjamin S, Andreas A, et al. LiveKrakenreal-time metagenomic classification of illumina data. Bioinformatics 2018;34:3750-2.

33. Xu XW, Wu XX, Jiang XG, et al. Clinical findings in a

Cite this article as: Li S, Li Z, Chen L, Cheng J, Zhan Y, Yang F, Jiang Q, Su X, Xie Y, Wu Z, Guo W, Gu W, Zhong N, Li S, Ye F. Epidemiological and clinical characteristics of suspected COVID-19 patients in the isolation ward in Guangzhou, China: a cohort study. Ann Transl Med 2021;9(8):621. doi: 10.21037/atm-20-6600 group of patients infected with the 2019 novel coronavirus (SARS-Cov-2) outside of Wuhan, China: retrospective case series. BMJ 2020;368:m606.

34. Sun S, Fu C, Cong J, et al. Epidemiological features and trends of influenza incidence in mainland China: A population-based surveillance study from 2005 to 2015 . Int J Infect Dis 2019;89:12-20.

35. Wrotek A, Czajkowska M, Jackowska T. Nosocomial Infections in Patients Hospitalized with Respiratory Syncytial Virus: A Practice Review. Adv Exp Med Biol 2020;1271:1-10.

36. Cheng Z, Lu Y, Cao Q, et al. Clinical Features and Chest CT Manifestations of Coronavirus Disease 2019 (COVID-19) in a Single-Center Study in Shanghai, China. AJR Am J Roentgenol 2020;215:121-6.

37. Yin S, Peng Y, Ren Y, et al. The implications of preliminary screening and diagnosis: Clinical characteristics of 33 mild patients with SARS-CoV-2 infection in Hunan, China. J Clin Virol 2020;128:104397.

38. Koo HJ, Lim S, Choe J, et al. Radiographic and CT Features of Viral Pneumonia. Radiographics 2018;38:719.

39. Ai T, Yang Z, Hou H, et al. Correlation of Chest CT and RT-PCR Testing for Coronavirus Disease 2019 (COVID-19) in China: A Report of 1014 Cases. Radiology 2020;296:E32-40.

40. Anderson RM, Hans H, Don K, et al. How will countrybased mitigation measures influence the course of the COVID-19 epidemic? Lancet (London, England) 2020;395:931-4. 
Supplementary

Table S1 Comparison of radiographic finding between the group

\begin{tabular}{|c|c|c|c|c|c|}
\hline Radiographic findings & $\begin{array}{l}\text { COVID-19 patients } \\
(n=23)\end{array}$ & $\begin{array}{l}\text { Other viral infection } \\
\text { patients }(n=30)\end{array}$ & $P$ value & $\begin{array}{l}\text { Nonviral infection } \\
\text { patients }(n=31)\end{array}$ & $P$ value \\
\hline Bilateral infiltrate on $\mathrm{CT}, \mathrm{n}(\%)$ & $22 / 23(95.7 \%)$ & $17 / 30(56.7 \%)$ & 0.04 & $15 / 31(48.4 \%)$ & 0.13 \\
\hline Single infiltrate on CT, n (\%) & $1 / 23(4.3 \%)$ & $13 / 30(43.3 \%)$ & 0.01 & $16 / 31(51.7 \%)$ & 0.03 \\
\hline Number of involved lobes on CT, median (range) & $4(1-5)$ & $2(1-5)$ & & $1(1-5)$ & \\
\hline \multicolumn{6}{|l|}{ Pattern of pulmonary infiltrates on CT, n (\%) } \\
\hline GGO only & $14 / 23(60.9 \%)$ & $20 / 30(66.7 \%)$ & 0.228 & $5 / 31(16.1 \%)$ & 0.04 \\
\hline GGO + consolidation & $9 / 23(39.1 \%)$ & $6 / 30(20.0 \%)$ & 0.07 & $3 / 31(9.6 \%)$ & 0.04 \\
\hline Consolidation & 0 & 0 & NA & $15 / 31(48.3 \%)$ & 0.015 \\
\hline
\end{tabular}

Not all the patients had finished the CT. COVID-19, coronavirus disease 2019; GGOs, ground-glass opacities. 